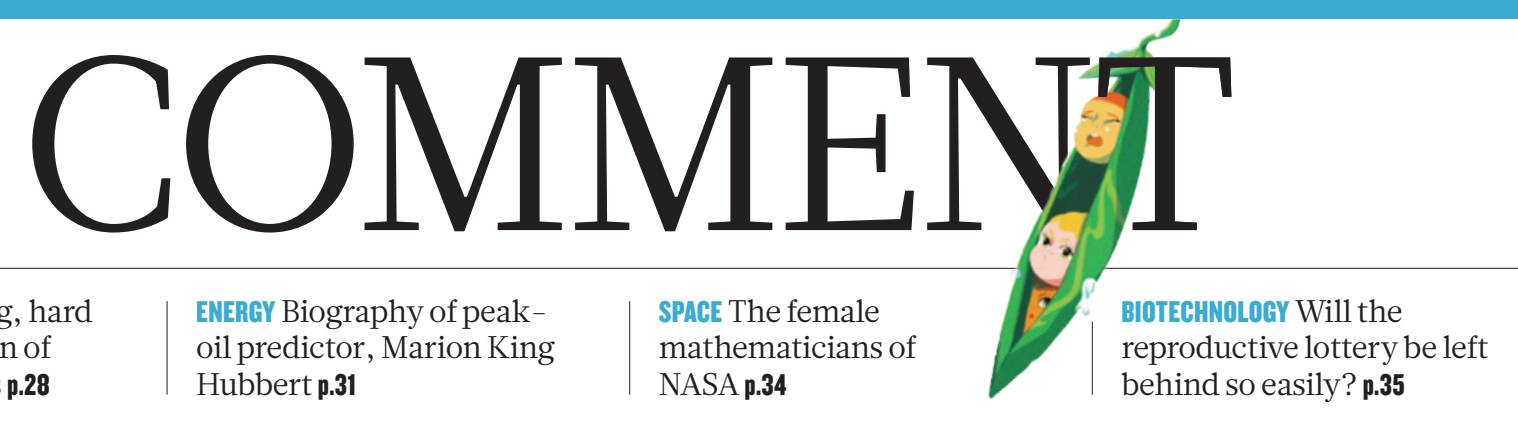

ASTROPHYSICS The long, hard road to the detection of gravitational waves p.28 oil predictor, Marion King Hubbert $\mathbf{p . 3 1}$
SPACE The female

NASA p.34 behind so easily? p.35

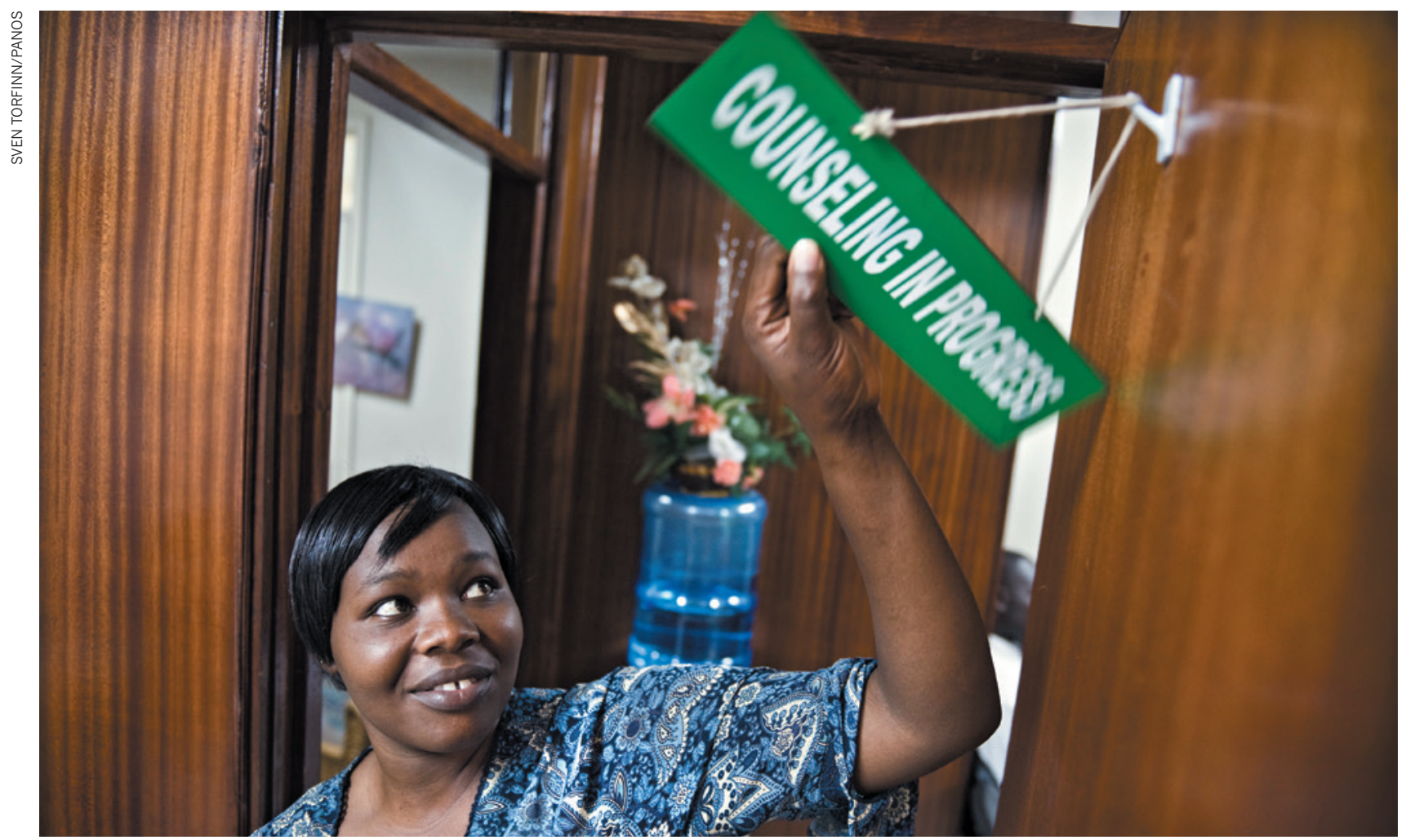

A counsellor at Nairobi Women's Hospital.

\title{
Action on mental health needs global cooperation
}

As threats to populations transcend national boundaries, it is crucial that responses to mental-health problems do too, say Pamela Y. Collins and Shekhar Saxena.

$\mathrm{M}$ ental-health disorders are the leading causes of disability worldwide. Nearly $30 \%$ of people around the world experience a mood, anxiety or substance-use disorder in their lifetime ${ }^{1}$. The resources required to address these conditions are inadequate, unequally distributed, inefficiently used and static ${ }^{2}$. The widespread incarceration of people with mental-health disorders persists.

The need and demand for mental-health care is increasing as vulnerable populations expand. Notable are the tens of millions of migrants fleeing persecution, conflict and violence, as well as the survivors of Ebola and other recent threats. Yet there are only 9 mental-health providers per 100,000 people globally; an extra 1.7 million mental-health workers are needed in lowand middle-income countries alone.

Mental health does not lack political support. This month, the World Bank and the World Health Organization (WHO) will together address the broader development community to make the case for investment in mental health. In the past three years, the importance of mental health has been highlighted by the WHO, in its Mental Health Action Plan for 2013-20; by leaders of countries in the Asia-Pacific Economic Cooperation (APEC); and by the health ministers of the Commonwealth nations. In September 2015, mental health was incorporated into the United Nations' Sustainable Development Goals.

Five years ago, we, as members of the 
- Grand Challenges in Global Mental Health initiative, called for an equitable and global approach to reducing the burden of mental disorders ${ }^{3}$. The visibility of the issue has come a long way since then. And although there continue to be problems with the delivery of mental-health services, funding for research and innovation in mental health in low- and middle-income countries has increased substantially (albeit from a small base). Since 2011, new investments estimated at US $\$ 79.3$ million have been made by the three largest funders of mental-health research in low- and middle-income countries (Grand Challenges Canada, the UK Department for International Development and the US National Institute of Mental Health).

Researchers in such countries are tackling the dearth of mental-health professionals by testing the delivery of care by non-specialists - such as peers, community health workers or primary-care providers. Others are developing and testing applications on smartphones and tablets to extend access to screening and treatment ${ }^{4}$ (see page 20).

Now, clinicians, patients, caregivers and researchers need to learn from each other. The knowledge gained in all countries must be evaluated, disseminated and adapted for local use everywhere. Crucially, everyone involved must start with the same mindset: when it comes to mental health, all countries are developing countries.

Of course, the resources available are drastically different in the developing and developed worlds: a teenager in Afghanistan seeking mental-health care does so in a country that has 1 psychiatrist for every 10 million people, not 1 per 5,000, as in, for instance, Belgium. But no country has sufficient numbers of trained mental-health-service providers. Nearly one-third of the US population lacks adequate access to mental-health-care providers. There are similar shortages in parts of countries as diverse as Australia, Canada, Finland, France, Japan, New Zealand and Slovakia. Even in wealthy countries, $40-60 \%$ of people with severe mental disorders do not receive the care they need ${ }^{5}$.

Across all settings, those with the fewest social and economic resources are least likely to receive quality mental-health services, be they in Arctic areas of Canada, innercity Glasgow or rural Sierra Leone. Today's global mental-health research must lead to interventions developed in and for such underserved communities.

\section{MOVING FORWARD}

Mirroring the global community's commitment to sustainable development, the world needs a global commitment - financial as well as moral - to mental health that supports the translation of ideas and interventions between poor and rich settings while taking into account local needs. If an

intervention shows great promise in Iran, for instance, can it be adapted for Poland or Indonesia?

In the 1980s and 1990s, global collaborative research led by the $\mathrm{WHO}$ enabled cross-national comparisons of the incidence, prevalence and long-term course of mental disorders, as well as cross-cultural conceptualizations of mental illness and traditional modes of understanding and management. Over the past 15 years, many of the efforts in global mental health have focused on introducing high-quality research in lowand middle-income countries to establish an evidence-base for the delivery of services in these nations.

More-recent research has focused on efficacy, effectiveness and implementation in low- and middle-income countries. Local research teams frequently collaborate with colleagues in rich countries. Yet, the relevance of this work to underserved populations in high-income countries is not routinely part of the global conversation. In low-income countries, the limited infrastructure for community mental-health care and the dismal budgetary allocations for mental health are significant obstacles to implementing research findings.

The status quo is not working - and innovations are needed urgently. The following case studies are exemplars of the approaches we advocate.

South-south learning. The Programme for Improving Mental Health Care (PRIME) is a consortium of research institutions and ministries of health funded by the UK government. PRIME aims to scale up mental-health services in Ethiopia, India, Nepal, South Africa and Uganda

by integrating these into primary care. Together, these countries have developed locally relevant mental-health plans informed by community advisory

"If an intervention shows great promise in Iran, for instance, can it be adapted for Poland or Indonesia?"

boards that include district health administrators, service users, traditional healers and police. The consortium observes crosscountry differences and similarities in the evolving mental-health-care systems.

The shared framework for developing and implementing plans with local adaptations is a powerful tool. Adaptations included change-management interventions for district managers in South Africa, a mental-health case manager in India, and new assessment tools in Nepal. All country teams have recognized the need for systemic changes. The next phase of the study is evaluation, to assess whether and how these changes affect disease burden.
North-north learning. The Arctic Council, an intergovernmental forum for the circumpolar states, has emerged as an avenue for launching collaborative efforts to reduce suicide rates in those countries. Young Alaska Native men experience the highest rates of suicide of any demographic group in the United States. Similarly high rates also occur among some indigenous Arctic communities in Canada, Greenland and Russia. Local responders can benefit from what has been learned and shown to be effective elsewhere.

An Arctic Council initiative that ran between 2013 and 2015, led by Canada, identified promising practices for suicide prevention and mental-health promotion, and mapped the evidence across circumpolar communities, noting what interventions were acceptable where. Teams identified common threads that made a programme scalable and adaptable across the region. These included having sustained funding and dedicated physical spaces for services; easy access for community members; having skilled workers who were based in and were knowledgeable about the community; and creating forums for talking about suicide. Crucially, the effort continues in the US-led RISING SUN initiative, which engages researchers, communitymembers and decision-makers to identify shared tools.

South-north learning. BasicNeeds is a global mental-health charity, established in 2000 in Britain, that facilitates access to employment and mental-health care for people with mental illness. The organization refined a model for helping people into care and work and to advocate for their problems in African and Asian countries, including Ghana, Tanzania, Nepal, China and Vietnam.

In Nepal, for example, a local charity that specialized in community-based rehabilitation adopted the BasicNeeds model. Working closely with government-funded mentalhealth clinics, the programme conducted community outreach and facilitated access to mental-health-care services. It reduced expenses for families with ill members. Eligible families received training and financial support for developing and implementing a business plan for income generation. People who received support were all earning money 6-12 months later. BasicNeeds received funding last year from the Robert Wood Johnson Foundation in Princeton, New Jersey, to translate the model to a deprived, inner-city environment in the United States.

This kind of translation of practices is just beginning. Technology is increasingly enabling innovators to make their ideas and projects public. One venue for sharing ideas is the Mental Health Innovation Network (MHIN), funded by Grand Challenges Canada and managed by a research team at the London School for Hygiene \& Tropical 


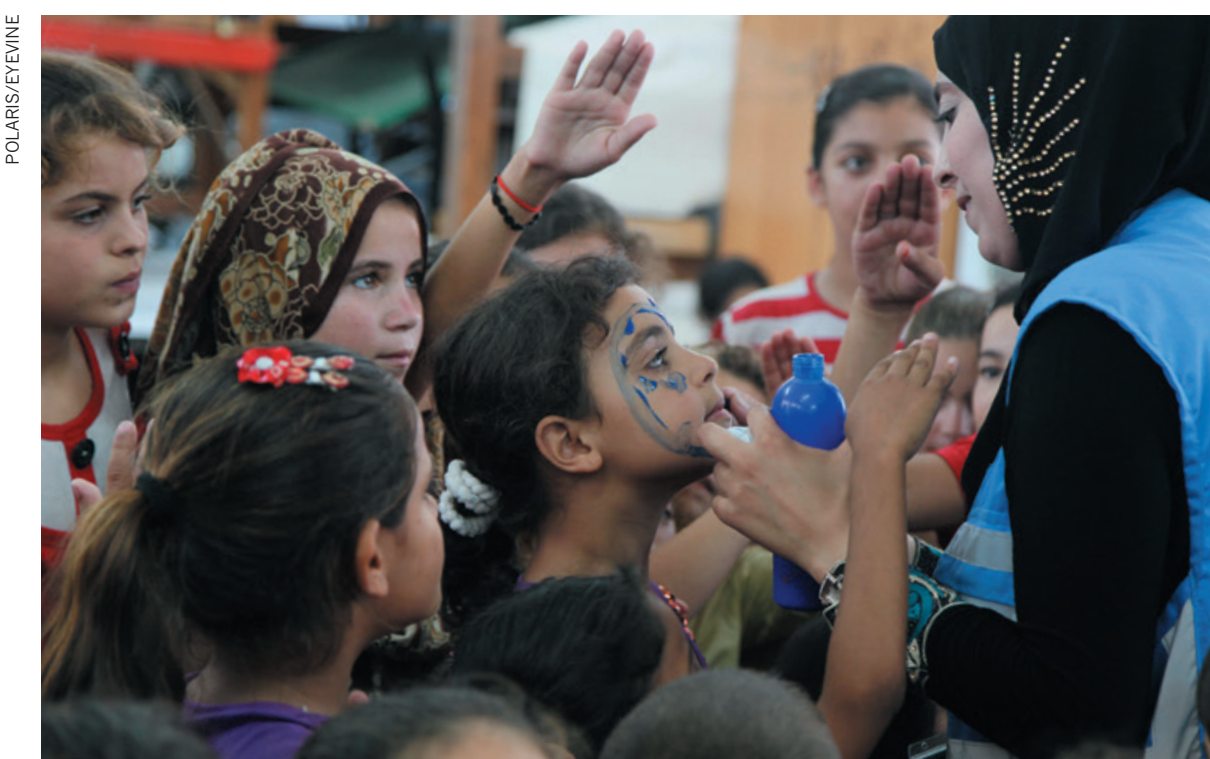

Palestinian children affected by conflict attend a UN mental-health programme in the Gaza Strip.

Medicine and the WHO's Department of Mental Health and Substance Abuse (of which S.S. is director). Another virtual community is the WHO's Global Clinical Practice Network. This online platform allows thousands of clinicians from around the world to contribute to and benefit from mental-health research. Through it, more than 12,000 clinicians from 139 countries have participated in field trials, testing diagnostic guidelines in a wide range of settings. Such networks also break national, professional and linguistic boundaries to facilitate global conversation and learning.

\section{NEXT STEPS}

To meet the mental-health needs of vulnerable people everywhere, we must develop, study and practise the translation of knowledge and ideas in all directions. How? Here are six suggestions.

Determine which innovations will scale up. Sometimes local application is enough. The community must distil guiding principles that enable practitioners to decide what is right for which contexts. This requires health planners to consider system-level issues (such as human resources and financing) and community-level needs (including acceptability and feasibility of care practices). In all contexts, cost, complexity and fragmented services can curtail wider implementation.

Train scientists to translate research findings. A new cadre of global mentalhealth researchers is needed to adapt treatments to fit local health systems. They must be able to assess needs and must be equipped with the collaborative skills to engage decision-makers, clinicians and community members. They need to generate knowledge that informs cross-cultural translation.
Use the community's knowledge. The growing evidence base on effective low-cost mental-health treatments is underused. Scientific knowledge is often inaccessible to practitioners, because they lack the time and resources to review information. We need to develop ways to synthesize new global mental-health findings routinely, and present this information so that users can apply it. The global fight against HIV/AIDS presents one model to draw from: networks of funders, researchers, clinicians and patients have been able to achieve standardized care protocols by sharing information through international working groups, society representatives and UNAIDS, the UN programme for HIV/AIDS. Similar networks exist in vaccine and contraception research.

Sustain effective mental-health treatments. A major problem is that research funding does not support continued delivery of services on the ground - this requires a greater commitment from local and national governments and aid agencies to invest in mental health. The WHO Mental Health Action Plan specifically calls for stronger leadership and governance for mental health at the national level, including adequate funding. Around $\$ 1.6$ billion is needed in low-income countries, and between $\$ 6.6$ billion and $\$ 9.33$ billion in lower-middle-income countries, to provide a basic package of mental health services; this is eight and six times more, respectively, than current investments ${ }^{6}$. The message that poor investment in mental health is costly for all countries must be communicated to leaders with the power to invest ${ }^{7}$.

Evaluate the outcomes of treatments. Globally, we lack adequate information on the impact of services because clinics and health systems often lack the funding, capacity, motivation and protocols for monitoring and evaluation. Rarer still is a mechanism for using the results of evaluation to improve services. So people need to be trained to monitor and evaluate new and established approaches. Collaborative research networks can facilitate this kind of capacity building. The WHO Mental Health Action Plan sets out six global targets to achieve by 2020. For example, it calls for a $20 \%$ increase in service coverage for severe mental disorders and a $10 \%$ reduction in suicide rates globally. Mental-health advocates, clinicians and patient groups in each country must track progress towards these targets.

Disseminate successes and failures. The risks that result from sharing information about programme weaknesses must be minimized. Researchers rely on journal publications to disseminate information, but it is much harder to publish unsuccessful trials or evaluations. We need options beyond research databases. Online platforms such as the MHIN could be used here, especially by those who are not researchers who develop new solutions to local problems.

In a world where mental-health innovations cross borders as people do, a mother migrating from Khayelitsha in South Africa to New York could meet a community health worker who delivers a depression treatment in her home, much like the community counsellor at her maternal health clinic in South Africa. People move because of needs and opportunities - so, too, must knowledge. @ SEE NEWS FEATURE P.20

Pamela Y. Collins is director of the Office for Research on Disparities \& Global Mental Health, US National Institute of Mental Health, Bethesda, Maryland, USA. Shekhar Saxena is director of the Department of Mental Health and Substance Abuse, World Health Organization, Geneva, Switzerland. e-mail:pamela.collins@nih.gov; saxenas@who.int

1. Steel, Z. et al. Int. J. Epidemiol. 43, 476-493 (2014).

2. Saxena, S., Thornicroft, G., Knapp, M. \& Whiteford, H. Lancet 370, 878-889 (2007).

3. Collins, P. Y. et al. Nature 475, 27-30 (2011).

4. Hamdani, S. U., Minhas, F. A., lqbal, Z. \& Rahman, A. Pediatrics 136, 1166-1172 (2015).

5. Wang, P. S. et al. Lancet 370, 841-850 (2007).

6. Gilbert, B. J., Patel, V., Farmer, P. E. \& Lu, C. PLoS Med. 12, e1001834 (2015).

7. Vigo, D., Thornicroft, G. \& Atun, R. Lancet Psychiatry 3, 171-178 (2016).

The views expressed here do not necessarily represent those of the US National Institute of Mental Health, the US National Institutes of Health, the US government or the World Health Organization. 